\title{
Pathogenicity Diversity in Albugo candida Isolates causing White Rust Disease in Rapeseed-Mustard
}

\author{
Devanshu Dev*, G. R. Daniel, Pooja Upadhyay and A. K. Tewari
}

Department of Plant Pathology, College of Agriculture, G B Pant University of Agriculture and Technology, Pantnagar-263145, Uttarakhand, India

*Corresponding author

\section{A B S T R A C T}

Keywords

Coalesced,

Pathogenicity

diversity, Oilseed

crop, Biotic stress,

White rust

Article Info

Accepted:

07 November 2019

Available Online:

10 December 2019
Albugo candida causing white rust of rapeseed-mustard is a highly variable fungus and cause great economic losses. In this study $44 \mathrm{~A}$. candida isolates belongs to 17 different states of India has been used for the pathogenicity diversity. Symptomatology of the pathogen i.e. pustule pattern, pustule size and pustule colour has taken as criteria for the pathogenicity diversity. All the 44 A. candida isolates were inoculated on susceptible Brassica juncea cultivar Varuna for the study. In the study of white rust symptoms of $44 A$. candida on Varuna cultivar five different types of pustule pattern has been observed viz. viz. Separate circular; Coalesced circular, Scattered and pin head; Restricted near veins and veinlets and Restricted near veins and veinlets separate or coalesced circular type pustules. Pustules size among $44 \mathrm{~A}$. candida isolates was varied from $0.5-2.5 \mathrm{~mm}$ while pustule colour of all the isolates was creamy white. The study very less pathogenicity diversity in pustule size and colour has been observed among $44 \mathrm{~A}$. candida isolates, while in pustule pattern some diversity was observed.

\section{Introduction}

Oilseed brassica is one of the most important oilseed crop of tropical and subtropical countries. Rapeseed-mustard affected by different biotic stress, among them white rust caused by $A$. candida is a major disease, causing heavy economic losses. It is estimated that the floral and leaves infection can cause losses from 20-60 per cent (Kolte, 2002; Khunti et al., 2003; Sachan et al., 2004; Kumar and Kalha, 2005) and up to 36.88 per cent (Bal and Kumar, 2014) in mustard. White rust symptoms are characterised as local and systemic. In case of local infection, symptoms of the disease appear primarily on the lower surface of leaves and manifested by the appearance of minute or small, white or 
creamy yellow raised scattered and circular pustules (1 to $2 \mathrm{~mm}$ in dia.) which later coalesce to form patches. Systemic infection symptoms include distortion, hypertrophy, hyperplasia and inflorescence sterility. This infection phase has been known as the stag head.

The flowers affected show malformation, the petals become green sepals and the stamens can be transformed into a leaf-like structure or a carpel.

The petals and stamens persist in the flower, as in normal flowers, rather than falling early. Sometimes the stamens become thick clubshaped sterile bodies. Usually the ovules and pollen grains are atrophied and result in incomplete sterility (Kolte, 1985). Yield loss is greater in case of systemic than local infection (Harper and Pittman, 1974; Petrie, 1973; Verma and Petrie, 1980). At maturity stage, stag heads are with thick-walled brown oospores that have been appeared to survive in dry form for a more than 20 years (Verma and Petrie, 1975).

A. candida isolates from different geographical regions and different host may be different in their incubation period, latent period and production of sporangia and zoospores, pustule size, shape and texture and aggressiveness (Lakra and Saharan, 1988; Gupta and Saharan, 2002; Patni et al., 2005 and Mishra et al., 2009). This indicated that there may be the possibilities of variability within A. candida isolates. The high variability of $A$. candida makes it a highly virulent pathogen, which is difficult to manage. The study of variability is needed to find out the status of variability, there occurrence and evolution pattern so that it will be helpful in the development of resistant varieties against the disease. Therefore, in this study pathogenicity variability of $44 \mathrm{~A}$. candida isolates has been done.

\section{Materials and Methods}

Forty four A. candida isolates collected from Oilseed Pathology Laboratory, Department of Plant Pathology were belonging to 17 states of India and also from 10 different Brassica spp. The description of $A$. candida isolates used in the studies is given in the table below (Table $1)$.

\section{Isolation and purification of white rust inoculum}

The isolation and purification of all $A$. candida isolates were done on susceptible $B$. juncea $\mathrm{cv}$. Varuna grown under glasshouse. The seedlings were raised in plastic pots containing sterilized soil. The fresh sporangial suspension was prepared with single pustule in a glass vial contained 1-2ml sterile distilled water. The suspension was mixed properly and kept it at $10^{\circ} \mathrm{C}$ for overnight for the release of zoospores. Although, freshly prepared sporangial suspension has also used for the inoculation without any incubation period. The sporangial concentration in the suspension was maintained about $2.5 \times 10^{5}$ zoosporangia per $\mathrm{ml}$ using haemocytometer. The seedlings at cotyledonary stage (7-8DAS) were inoculated with $10 \mu \mathrm{l}$ spore suspension through inoculating micropipette on each and every lobes of cotyledon of the seedlings. After inoculation plants were transfer to plant propagator box and kept the box in a dark room for $72 \mathrm{hrs}$, with a relative humidity of more than 90 per cent and of $18 \pm 2{ }^{\circ} \mathrm{C}$ temperature. After three days plants were taken out and kept in glasshouse where, proper temperature $\left(18 \pm 2^{\circ} \mathrm{C}\right)$ and humidity $(>80 \%)$ were maintained during the entire plant growth and disease appearance period. When disease appeared (10-15DAI), infected leave samples were collected before yellowing of the leaves and preserved at low temperature $\left(4^{\circ} \mathrm{C}-10^{\circ} \mathrm{C}\right)$ by 
keeping it in butter paper bags for further studies. The pustule pattern of all $44 A$. candida isolates were observed and recorded. Likewise, pustule dia. of 10 randomly selected leaves of an isolate were taken in mm. Analysis of mean pustule size of all $44 \mathrm{~A}$. candida isolates has been done through Duncan's Multiple Range Test (DMRT) using SPSS 16.0 version software to find out whether there is significant difference between the mean pustule size. Pustule colour in symptomatic leaves of each isolate was observed and recorded.

\section{Results and Discussion}

The pathogenicity diversity of 44 A. candida isolates has been done on the $B$. juncea cv. Varuna. The colour of pustules of all the isolates was creamy white in colour. The pustule pattern of all the isolates has been observed visually and categorized in 05 different types of pustule pattern viz. Separate circular; Coalesced circular, Scattered and pin head; Restricted near veins and veinlets and Restricted near veins and veinlets separate or coalesced circular type pustules.

Nine showed separate circular type, 16 of coalesced circular type, 12 of scattered pin head type, 04 of restricted near vein and veinlet type and 03 of restricted near vein and veinlet separate or coalesced circular type of pustule (Table 2).

The variation in pustule pattern of $33 \mathrm{~A}$. candida isolate indicated different level of pathogenicity diversity. The isolates that formed different pustule pattern could be of different pathotypes. The similar type of observations has been reported by Pandey et al., (2013) where pinhead raised pustules were observed in isolates of Pantnagar, Delhi, Bharatpur, Hisar, Kangra, and Jammu, while pinhead scattered pustules in isolates of Ludhiana. Based on pustules pattern 33 A. candida isolates were grouped in 05 pathotypes.

In Duncan's multiple range test (DMRT) of mean pustule size of $A$. candida isolates varied from $0.5-2.5 \mathrm{~mm}$ in dia. The lowest mean pustule size i.e. $0.5 \mathrm{~mm}$ dia. was observed in Ac-MMP (B. juncea cv. Maya, Morena, MP) while, highest mean pustule size i.e. $2.5 \mathrm{~mm}$ dia. was observed in Ac-BjV (B. juncea cv. Varuna, Pantnagar, Uttarakhand), Ac-Knp (Kanpur, UP) etc. The mean pustule size was $1.59 \mathrm{~mm}$ dia. In DMRT of mean pustule size of 44 A. candida isolates, less variability has been observed (Table 3 ).

The similar type of observations has been reported by Pandey et al., (2013) the small size pustules $(0.5-1.5 \mathrm{~mm})$ was observed in New Delhi and Hisar isolates of A. candida, while large size pustules (1-3 $\mathrm{mm})$ in Pantnagar and Ludhiana isolates of $A$. candida.

In this study pathogenicity diversity of $A$. candida isolates has been observed based on symptomatological observations. The significant diversity has been observed in pustule pattern of different $A$. candida isolates which could be utilized for the further study. While, in case of pustule colour and pustule size of $A$. candida isolates, there was no significant difference observed. 
Table.1

\begin{tabular}{|c|c|c|c|c|c|}
\hline S.No. & $\begin{array}{l}\text { Isolate } \\
\text { Name }\end{array}$ & Host & Place & State & $\begin{array}{l}\text { Latitude \& } \\
\text { Longitude }\end{array}$ \\
\hline 1. & Ac-BjV & B. juncea cv. Varuna & \multirow[t]{10}{*}{ Pantnagar } & \multirow[t]{10}{*}{ Uttarakhand } & \multirow{10}{*}{$\begin{array}{l}29^{\circ} 02^{\prime} 60^{\prime \prime} \mathrm{N} \\
79^{\circ} 30^{\prime} 59^{\prime \prime} \mathrm{E}\end{array}$} \\
\hline 2. & Ac-Brai & Banarasi rai & & & \\
\hline 3. & Ac-Brys & B. rapa yellow sarson & & & \\
\hline 4. & Ac-BjBio & B. juncea cv.Bio-YSR & & & \\
\hline 5. & $\mathrm{Ac}-\mathrm{Br}$ & B. rugose & & & \\
\hline 6. & Ac-Bni & B. nigra & & & \\
\hline 7. & Ac-Bna & B. napus & & & \\
\hline 8. & Ac-BjK & B. juncea cv.Kranti & & & \\
\hline 9. & Ac-Orai & Ornamental rai & & & \\
\hline 10. & Ac-Kw & Kattili weed & & & \\
\hline 11. & Ac-Knp & B. juncea & Kanpur & \multirow[t]{3}{*}{ Uttar Pradesh } & $\begin{array}{l}26^{\circ} 26^{\prime} 59.6^{\prime \prime} \mathrm{N} \\
80^{\circ} 19^{\prime} 54.8^{\prime \prime} \mathrm{E}\end{array}$ \\
\hline 12. & Ac-Met & B. juncea & Meerut & & $\begin{array}{r}28^{\circ} 59^{\prime} 4^{\prime \prime} \mathrm{N} \\
77^{\circ} 42^{\prime} 21^{\prime \prime} \mathrm{E}\end{array}$ \\
\hline 13. & Ac-Fzb & B. juncea & Faizabad & & $\begin{array}{l}26^{\circ} 46^{\prime} 12^{\prime \prime} \mathrm{N} \\
82^{\circ} 9^{\prime} 0.00^{\prime \prime} \mathrm{E}\end{array}$ \\
\hline 14. & Ac-Alw & B. juncea & Alwar & \multirow[t]{4}{*}{ Rajasthan } & $\begin{array}{l}27^{\circ} 33^{\prime} 39^{\prime \prime} \mathrm{N} \\
76^{\circ} 37^{\prime} 30^{\prime \prime} \mathrm{E}\end{array}$ \\
\hline 15. & Ac-Bhrtp & B. juncea & Bharatpur & & $\begin{array}{c}27^{\circ} 13^{\prime} 11^{\prime \prime} \mathrm{N} \\
77^{\circ} 29^{\prime} 22^{\prime \prime} \mathrm{E}\end{array}$ \\
\hline 16. & Ac-Kot & B. juncea & Kotputli & & $\begin{array}{l}27^{\circ} 42^{\prime} 13^{\prime \prime} \mathrm{N} \\
76^{\circ} 12^{\prime} 4^{\prime \prime} \mathrm{E}\end{array}$ \\
\hline 17. & Ac-Sgn & B. juncea & $\begin{array}{c}\text { Sri } \\
\text { Ganganagar }\end{array}$ & & $\begin{array}{l}29^{\circ} 54^{\prime} 13^{\prime \prime} \mathrm{N} \\
73^{\circ} 52^{\prime} 37^{\prime \prime} \mathrm{E}\end{array}$ \\
\hline 18. & Ac-Knc & B. juncea & Kannahalli & \multirow[t]{2}{*}{ Karnataka } & $\begin{array}{l}12^{\circ} 52^{\prime} 15^{\prime \prime} \mathrm{N} \\
76^{\circ} 47^{\prime} 18^{\prime \prime} \mathrm{E}\end{array}$ \\
\hline 19. & Ac-Bnglr & B. juncea & Bangalore & & $\begin{array}{l}12^{\circ} 58^{\prime} 20^{\prime \prime} \mathrm{N} \\
77^{\circ} 34^{\prime} 50^{\prime \prime} \mathrm{E}\end{array}$ \\
\hline 20. & Ac-Ldh & B. juncea & Ludhiana & Punjab & $\begin{array}{r}30^{\circ} 54^{\prime} 3^{\prime \prime} \mathrm{N} \\
75^{\circ} 51^{\prime} 26^{\prime \prime} \mathrm{E}\end{array}$ \\
\hline 21. & Ac-Abr & B. juncea & Abohar & & $\begin{array}{l}30^{\circ} 08^{\prime} 40^{\prime \prime} \mathrm{N} \\
74^{\circ} 11^{\prime} 43^{\prime \prime} \mathrm{E}\end{array}$ \\
\hline 22. & Ac-VND & B. juncea cv. Varuna & IARI & \multirow[t]{4}{*}{ New Delhi } & $28^{\circ} 37^{\prime} 55^{\prime \prime} \mathrm{N}$ \\
\hline 23. & Ac-PbND & B. juncea cv. PusaBahar & IARI & & $77^{\circ} 8^{\prime} 19^{\prime \prime} \mathrm{E}$ \\
\hline 24. & $\mathrm{Ac}-\mathrm{BcND}$ & B. carinata cv. BCS-1 & IARI & & \\
\hline 25. & Ac-UD & B. juncea & UDSC & & $\begin{array}{c}28^{\circ} 38^{\prime} 41^{\prime \prime} \mathrm{N} \\
77^{\circ} 13^{\prime} 0^{\prime \prime} \mathrm{E}\end{array}$ \\
\hline 26. & Ac-Hsr & B. juncea & Hisar & \multirow[t]{3}{*}{ Haryana } & $\begin{array}{l}29^{\circ} 9^{\prime} 6.69^{\prime \prime} \mathrm{N} \\
75^{\circ} 43^{\prime} 16^{\prime \prime} \mathrm{E}\end{array}$ \\
\hline 27. & Ac-Krnl & B. juncea & Karnal & & $\begin{array}{c}29^{\circ} 41^{\prime} 8.2^{\prime \prime} \mathrm{N} \\
76^{\circ} 59^{\prime} 25^{\prime \prime} \mathrm{E}\end{array}$ \\
\hline 28. & Ac-Srsa & B. juncea & Sirsa & & $\begin{array}{c}29^{\circ} 32^{\prime} 11^{\prime \prime} \mathrm{N} \\
75^{\circ} 1^{\prime} 31^{\prime \prime} \mathrm{E}\end{array}$ \\
\hline 29. & Ac-BR & B. juncea & Pusa, & Bihar & $25^{\circ} 51^{\prime} 39^{\prime \prime} \mathrm{N}$ \\
\hline
\end{tabular}




\begin{tabular}{|c|c|c|c|c|c|}
\hline & & & Samastipur & & $85^{\circ} 46^{\prime} 56^{\prime \prime} \mathrm{E}-$ \\
\hline 30. & Ac-Kng & B. juncea & Kangra & \multirow[t]{3}{*}{$\begin{array}{l}\text { Himachal } \\
\text { Pradesh }\end{array}$} & $\begin{array}{l}32^{\circ} 5^{\prime} 59^{\prime \prime} \mathrm{N} \\
76^{\circ} 16^{\prime} 8^{\prime \prime} \mathrm{E}\end{array}$ \\
\hline 31. & Ac-Smr & B. juncea & Sirmaur & & $\begin{array}{l}30^{\circ} 38^{\prime} 24^{\prime \prime} \mathrm{N} \\
77^{\circ} 26^{\prime} 24^{\prime \prime} \mathrm{E}\end{array}$ \\
\hline 32. & Ac-Psb & B. juncea & Ponta Sahib & & $\begin{array}{l}30^{\circ} 26^{\prime} 16^{\prime \prime} \mathrm{N} \\
77^{\circ} 37^{\prime} 26^{\prime \prime} \mathrm{E}\end{array}$ \\
\hline 33. & Ac-PbMP & B. juncea cv. Pusa bold & \multirow[t]{4}{*}{ Morena } & \multirow{4}{*}{$\begin{array}{l}\text { Madhya } \\
\text { Pradesh }\end{array}$} & \multirow{4}{*}{$\begin{array}{l}26^{\circ} 30^{\prime} 0.0^{\prime \prime} \mathrm{N} \\
78^{\circ} 0^{\prime} 0.00^{\prime \prime} \mathrm{E}\end{array}$} \\
\hline 34. & Ac-VdMP & B. junceacv. Vardan & & & \\
\hline 35. & Ac-MMP & B. junceacv. Maya & & & \\
\hline 36. & Ac-VMP & B. junceacv. Varuna & & & \\
\hline 37. & Ac-Jam & B. juncea & Chatha & $\begin{array}{c}\text { Jammu \& } \\
\text { Kashmir }\end{array}$ & $\begin{array}{l}32^{\circ} 43^{\prime} 58^{\prime \prime} \mathrm{N} \\
74^{\circ} 51^{\prime} 51^{\prime \prime} \mathrm{E}\end{array}$ \\
\hline 38. & Ac-Meg & B. juncea & Umiam & Meghalaya & $\begin{array}{c}25^{\circ} 34^{\prime} 0.1^{\prime \prime} \mathrm{N} \\
91^{\circ} 52^{\prime} 59^{\prime \prime} \mathrm{E}\end{array}$ \\
\hline 39. & Ac-WB & B. juncea & Kolkata & \multirow[t]{2}{*}{ West Bengal } & $22^{\circ} 34^{\prime} 21^{\prime \prime} \mathrm{N}$ \\
\hline 40. & Ac-AWB & Amaranthus & Kolkata & & $88^{\circ} 21^{\prime} 50^{\prime \prime} \mathrm{E}$ \\
\hline 41. & Ac-Asm & B. juncea & Jorhat & Assam & $\begin{array}{l}26^{\circ} 45^{\prime} 28^{\prime \prime} \mathrm{N} \\
94^{\circ} 12^{\prime} 35^{\prime \prime} \mathrm{E}\end{array}$ \\
\hline 42. & Ac-MZ & B. juncea & Aizawl & Mizorum & $\begin{array}{l}23^{\circ} 43^{\prime} 37^{\prime \prime} \mathrm{N} \\
92^{\circ} 43^{\prime} 3.4^{\prime \prime} \mathrm{E}\end{array}$ \\
\hline 43. & Ac-Mnp & B. juncea & Imphal & Manipur & $\begin{array}{l}24^{\circ} 48^{\prime} 50^{\prime \prime} \mathrm{N} \\
93^{\circ} 57^{\prime} 1.0^{\prime \prime} \mathrm{E}\end{array}$ \\
\hline 44. & Ac-SK & B. juncea & Marchak & Sikkim & $\begin{array}{l}27^{\circ} 19^{\prime} 48^{\prime \prime} \mathrm{N} \\
88^{\circ} 37^{\prime} 12^{\prime \prime} \mathrm{E}\end{array}$ \\
\hline
\end{tabular}

Table.2 Grouping of A. candida isolates based on pustule pattern

\begin{tabular}{|l|c|c|}
\hline Group & Pustules Pattern & A. candidaisolate \\
\hline $\mathbf{1 .}$ & $\begin{array}{c}\text { Separate and circular } \\
\text { type pustules }\end{array}$ & $\begin{array}{r}\text { Ac-Brai, Ac-Brys, Ac-Bna, Ac-Orai, Ac-Met,Ac- } \\
\text { Alw,Ac-Bhrtp, Ac-Ldh, Ac-VND }\end{array}$ \\
\hline 2. & $\begin{array}{c}\text { Coalesced circular type } \\
\text { pustules }\end{array}$ & $\begin{array}{c}\text { Ac-BjV, Ac-BjK, Ac-Br,Ac-Kw, Ac-Knp, Ac-BcND, } \\
\text { Ac-Hsr, Ac-Krnl, Ac-BR, Ac-Kng, Ac-PbMP, Ac- } \\
\text { VMP, Ac-MMP, Ac-Jam, Ac-Meg, Ac-Mnp }\end{array}$ \\
\hline 3. & $\begin{array}{c}\text { Scattered, pin head type } \\
\text { pustules }\end{array}$ & $\begin{array}{c}\text { Ac-BjBio, Ac-Bni, Ac-Knc, Ac-Bnglr, Ac-PbND, Ac- } \\
\text { Smr, Ac-WB,Ac-AWB, Ac-Sgn, Ac-Asm, Ac-MZ, } \\
\text { Ac-SK }\end{array}$ \\
\hline 4. & $\begin{array}{c}\text { Restricted near veins and } \\
\text { veinlets type Pustules } \\
\text { Restricted near veins and } \\
\text { veinlets separate or } \\
\text { coalesced circular type } \\
\text { Pustules }\end{array}$ & Ac-Fzb, Ac-Psb, Ac-VMP, Ac-UD \\
\hline 5. & Ac-Abr, Ac-Srsa, Ac-Kot \\
\hline
\end{tabular}


Table.3 Duncan's multiple range tests for mean pustule diameter

\begin{tabular}{|c|c|c|}
\hline Sr. No. & A. candida isolate & Average pustule size (mm) \\
\hline 1 & Ac-BjV & $2.5^{\mathrm{c}}$ \\
\hline 2 & Ac-Brai & $1.0^{\mathrm{ab}}$ \\
\hline 3 & Ac-Brys & $1.5^{\mathrm{abc}}$ \\
\hline 4 & Ac-BjBio & $1.5^{\mathrm{abc}}$ \\
\hline 5 & $\mathrm{Ac}-\mathrm{Br}$ & $1.5^{\mathrm{abc}}$ \\
\hline 6 & Ac-Bni & $2.0^{\mathrm{bc}}$ \\
\hline 7 & Ac-Bna & $2.0^{\mathrm{bc}}$ \\
\hline 8 & Ac-BjK & $1.5^{\mathrm{abc}}$ \\
\hline 9 & Ac-Orai & $1.0^{\mathrm{ab}}$ \\
\hline 10 & Ac-Kw & $1.5^{\mathrm{abc}}$ \\
\hline 11 & Ac-Knp & $2.5^{\mathrm{c}}$ \\
\hline 12 & Ac-Met & $2.5^{\mathrm{c}}$ \\
\hline 13 & Ac-Fzb & $1.0^{\mathrm{ab}}$ \\
\hline 14 & Ac-Alw & $2.0^{\mathrm{bc}}$ \\
\hline 15 & Ac-Bhrtp & $2.0^{\mathrm{bc}}$ \\
\hline 16 & Ac-Kot & $1.0^{\mathrm{ab}}$ \\
\hline 17 & Ac-Sgn & $0.8^{\mathrm{a}}$ \\
\hline 18 & Ac-Knc & $2.5^{\mathrm{c}}$ \\
\hline 19 & Ac-Bnglr & $2.5^{\mathrm{c}}$ \\
\hline 20 & Ac-Ldh & $2.0^{\mathrm{bc}}$ \\
\hline 21 & Ac-Abr & $1.0^{\mathrm{ab}}$ \\
\hline 22 & Ac-VND & $1.0^{\mathrm{ab}}$ \\
\hline 23 & $\mathrm{Ac}-\mathrm{PbND}$ & $1.0^{\mathrm{ab}}$ \\
\hline 24 & Ac-BcND & $1.0^{\mathrm{ab}}$ \\
\hline 25 & Ac-UD & $2.5^{\mathrm{c}}$ \\
\hline 26 & Ac-Hsr & $2.5^{\mathrm{c}}$ \\
\hline 27 & Ac-Krnl & $1.0^{\mathrm{ab}}$ \\
\hline 28 & Ac-Srsa & $0.5^{\mathrm{a}}$ \\
\hline 29 & Ac-BR & $2.5^{\mathrm{c}}$ \\
\hline 30 & Ac-Kng & $1.5^{\mathrm{abc}}$ \\
\hline 31 & Ac-Smr & $2.5^{\mathrm{c}}$ \\
\hline 32 & Ac-Psb & $1.0^{\mathrm{ab}}$ \\
\hline 33 & Ac-PbMP & $2.0^{\mathrm{bc}}$ \\
\hline 34 & Ac-VdMP & $1.0^{\mathrm{ab}}$ \\
\hline 35 & Ac-MMP & $0.5^{\mathrm{a}}$ \\
\hline 36 & Ac-VMP & $2.0^{\mathrm{bc}}$ \\
\hline 37 & Ac-Jam & $2.5^{\mathrm{c}}$ \\
\hline 38 & Ac-Meg & $1.0^{\mathrm{ab}}$ \\
\hline 39 & Ac-WB & $2.0^{\mathrm{bc}}$ \\
\hline 40 & Ac-AmWB & $2.0^{\mathrm{bc}}$ \\
\hline 41 & Ac-Asm & $0.8^{\mathrm{a}}$ \\
\hline 42 & Ac-MZ & $0.8^{\mathrm{a}}$ \\
\hline 43 & Ac-Mnp & $1.0^{\mathrm{ab}}$ \\
\hline 44 & Ac-SK & $1.5^{\mathrm{abc}}$ \\
\hline \multicolumn{2}{|r|}{ Mean } & 1.59 \\
\hline \multicolumn{2}{|r|}{ SEM } & 0.07 \\
\hline
\end{tabular}




\section{References}

Bal, R.S., Kumar, K. and Singh, P. 2014. Epidemiology and management of white rust and Alternaria blight of Indian mustard. Agric. Res. J. 51: 146149.

Gupta, K. and Saharan, G.S. 2002. Identification of pathotypes of Albugo candida with stable characteristic symptoms on Indian mustard. $J$. Mycol. Pl. Pathol. 32: 46-51.

Harper, F.R. and Pittman, U.J. 1974. Yield loss by Brassica campestris and Brassica napus from systemic stem infection by Albugo curciferamm. Phytopathol. 64: 408-410.

Khunti, J.P., Khandar, P.R. and Bhoraniya, M.F. 2003. Field evaluation of mustard (Brassica juncea L.) genotypes against white rust (Albugo cruciferarum S.F. Gray). Agric. Sci. Dig. 23:57-58.

Kolte, S.J. 1985. Diseases of annual edible oilseed crops. Volume II: Rapeseed mustard and sesame diseases. CRC Press, Inc., Boca Raton, Florida. $135 \mathrm{p}$

Kolte, S.J. 2002. Diseases and their management in oilseed crops. In Rai, M., Singh, H. and Hedge, D.M. (Eds.), New paradigm in oilseeds and oils: research and development needs (pp. 244-252). Hyderabad, India: Indian Society of Oilseeds Research.

Kumar, S., and Kalha, C.S. 2005. Evaluation of rapeseed-mustard germplasm against white rust and Alternaria blight. Ann. Biol. 21: 73-77.

Lakra, B.S. and Saharan, G.S. 1988.
Morphological and pathological variations in Albugo candida associated with Brassica species. Indian J. Mycol. Pl. Path. 18:149-156.

Mishra, K.K., Kolte, S.J., Nashaat, N.I. and Awasthi, R.P. 2009. Pathological and biochemical changes in Brassica juncea (mustard) infected with Albugo candida (white rust). Pl. Pathol. 58: 80-86.

Pandey, P., Tewari, A.K. and Awasthi, R.P. 2013. Morphological and pathogenic variability of Albugo candida isolates causing white rust in rapeseedmustard. The Bioscan 8(3):835-838.

Patni, C.S., Singh, A., and Awasthi, R.P. 2005. Variability in Albugo candida causing white rust disease of rapeseedmustard. J. Res. SKUAST-J. 4: 184191.

Petrie, G.A. 1973. Diseases of Brassica species in Saskatchewan, 1970-72. I. Staghead and aster yellows. Can. Plant Dis. Surv. 53: 19-25.

Sachan, J.N., Singh, A., Kolte, S.J., Prasad, L. and Singh, B. 2004. Evaluation of mustard germplasm against Albugo candida. Cruciferae Newsletter. 25: 87-88.

Verma, P.R. and Petrie, G.A. 1975. Germination of oospores of $A$. candida. Can. J. Bot. 53: 836-842.

Verma, P.R. and Petrie, G.A. 1980. Effect of seed infestation and flower bud inoculation on systemic infection of turnip rape by $A$. candida. Can. J. Plant Sci. 60: 267-271.

\section{How to cite this article:}

Devanshu Dev, G. R. Daniel, Pooja Upadhyay and Tewari, A. K. 2019. Pathogenicity Diversity in Albugo candida Isolates causing White Rust Disease in Rapeseed-Mustard. Int.J.Curr.Microbiol.App.Sci. 8(12): 621-627. doi: https://doi.org/10.20546/ijcmas.2019.812.081 